\section{Kopfschmerz und}

\section{Adhärenz bei der Verwen- dung einer Tagebuch-App}

*** Seng EK et al. Anxiety, Incentives, and Adherence to Self-Monitoring on a Mobile Health Platform: A Naturalistic Longitudinal Cohort Study in People With Headache. Headache 2018; doi: $10.1111 /$ head. 13422 .

Adhärenz stellt auch bei einer Kopfschmerztagebuch-App eine Herausforderung dar.

\section{Zusammenfassung}

Im therapeutischen Alltag empfiehlt sich das Führen von Kopfschmerztagebüchern, um Diagnosen besser spezifizieren und therapeutische Effekte besser einschätzen zu können. Eine geringe Adhärenz birgt die Gefahr falscher Diagnosen und das Übersehen schmerzbegünstigender Faktoren. Daten zur Adhärenz bei klassischen Tagebüchern stammen aus kleinen Studien und ergeben ein unklares Bild; mittels Apps können größere Datensätze gewonnen werden.

Die Autoren haben in einer naturalistischen Beobachtungsstudie die Tagebuchfunktion der App Curelator ${ }^{\circledR}$ bei 1561 Kopfschmerzpatienten hinsichtlich verschiedener Adhärenzmaße für einen Zeitraum von mind. 90 Tagen untersucht. Dies waren (a) vollständiges Ausfüllen von 90 Tagen, (b) Adhärenzrate (Anteil an ausgefüllten Tagen 90 Tage nach Registrierung) und (c) verzögertes Ausfüllen (Anzahl der Tage nach 90 Tagen, um 90 ausgefüllte Tage zu erreichen). Ein Teil der Patienten verwendete die Bezahlversion der App ( $8 \%$ ), ein Teil eine Couponversion ( $18 \%$, der Coupon wurde durch den behandelnden Arzt ausgegeben, nur nach vollständigem Ausfüllen erhielten die Patienten Auswertediagramme), und der größte Teil die kostenlose Version (74\%).

90 \% gaben Migräne als Kopfschmerz mit ungefähr 10 Kopfschmerztagen pro Monat an. Ein Drittel der Patienten erreichte (a) vollständiges Ausfüllen von insg. 90 Tagen (ca. 14\% nach 90 Tagen). Höhere tägliche Angstwerte gingen mit einem geringeren, höheres Alter mit einer höheren Odds Ratio für vollständiges Ausfüllen einher. Bei den Versionen gab es höhere Odds Ratios für Bezahlversion vs. Coupon, Bezahlversion vs. kostenlos und Coupon vs. kostenlos. (b) Vergleichbare Ergebnisse fanden sich für die Adhärenzrate. Höhere Angst und geringes Alter gingen mit geringerer Adhärenzrate einher, ebenso die Verwendung der kostenlosen Version. (c) Die meisten Teilnehmer die 90 Tage ausfüllten, taten dies innerhalb von 120 Tagen. Höhere Angst, geringeres Alter und die kostenlose Version waren mit längerer Verzögerung assoziiert.

Die Autoren diskutieren die Ergebnisse hinsichtlich der erfassten Einflussfaktoren, z. B. dass gebotene Anreize bzw. die Registrierung der Adhärenz (wie in der Couponversion) mit einer höheren Adhärenz einhergehen, dass finanzielle Ausgaben (wie in der Bezahlversion) im Sinne der sozialen Austauschtheorie die Motivation erhöhen könnte. Limitationen werden aufgezählt., z. B. erlaubt die Studie keine kausalen Aussagen; Kopfschmerz wurde nicht durch einen Arzt diagnostiziert; die Verwendung nur einer spezifischen App mit bestimmten Merkmalen lässt sich nicht auf andere Apps übertragen; Angst wurde nur über eine Frage erfasst „Wie nervös/ängstlich/ besorgt haben Sie sich heute gefühlt?“.

\section{Kommentar}

Die Studie hat sich Fragen angenommen, die in der Zeit zunehmender Digitalisierung in der Medizin von Relevanz sind. Wie sieht es mit der Adhärenz bei Kopfschmerztagebuch-Apps aus? Welche Einflussfaktoren gibt es? Zusammenfassend waren höhere Angst, geringeres Alter und die Verwendung der kostenlosen Version mit reduzierter Adhärenz assoziiert. Die Adhärenzergebnisse lassen sich - auch wenn in den Studien nicht immer vergleichbare Maße erfasst werden - in die bestehende Literatur einordnen. Dies zeigt, dass die digitale Erhebung von Kopfschmerzfragebuchdaten grundsätzlich möglich ist.

Interessant ist für den therapeutischen Einsatz vor allem, welche Faktoren die Adhärenz beeinflussen; wobei von kausalen Überlegungen abgesehen werden sollte. Das Setzen von bestimmten Anreizen, aber auch die kontinuierliche Registrierung („Überwachung“) von Patientenverhalten könnten therapeutische Möglichkeiten bieten. Therapeuten sollten immer auf Angstsymptome achten, da diese die Adhärenz reduzieren können. In der Studie wurde Angst relativ unspezifisch erfasst, eine detaillierte Erfassung wäre wünschenswert. Bemerkenswert ist, dass die Adhärenz bei kostenlosen Apps am geringsten zu sein scheint. Die Gründe wurden nicht erfragt; Patienten könnten hier eine geringere Qualitätssicherung vermuten bzw. bei der Bezahlversion besonders motiviert sein. Im Rahmen der Einführung zukünftiger medizinischer Apps sollte dies berücksichtigt werden. Insbesondere die vermittelnden psychologisch relevanten, motivationalen Variablen sollten weiter untersucht werden. So wäre es aufschlussreich gewesen, die Gründe für das komplette Ausfüllen zu erfragen. Dies sind Fragen, die unabhängig vom App-Einsatz gestellt und beantwortet werden müssen, um mögliche Subgruppen mit zu erwartender reduzierter Adhärenz identifizieren zu können.

Offen bleibt, inwiefern Kopfschmerztagebücher über Apps realisiert werden sollten. Die Erfassung im Forschungskontext hat zunächst den Vorteil, größere Datenmengen zu sammeln und bessere, robustere Analysemethoden einzusetzen. Sind Ergebnisse für die klassische und die App-Variante vergleichbar, kann die Wahl für oder wider erfolgen. Der Einsatz von Apps dürfte dann insgesamt von verschiedenen Präferenzen auf Patienten- und Therapeutenseite abhängen, welche im therapeutischen Setting und Prozess berücksichtigt werden müssen .

Thomas Dresler, Tübingen

\section{Migräne und Nacken- muskulatur}

\footnotetext{
** Luedtke K, Mehnert J, May A. Altered muscle activity during rest and during mental or physical activity is not a trait symptom of migraine. J Headache Pain 2018; 19: 26.
}

Bei Patienten mit Migräne zeigt die EMG-Aktivität im Musculus trapecius in Ruhe sowie bei geistiger und körperlicher Aktivität - mit einer Ausnahme unter 8 Bedingungen - keinen Unterschied zu migränefreien Personen. 


\section{Zusammenfassung}

Nackenschmerzen sind ein häufiges Phänomen bei Migräne. Ob sie Auslöser oder Symptom einer Attacke sind, und wie sie mit EMG-Befunden korrelieren, wird kontrovers diskutiert. Die Autorlnnen versuchten diese Frage mit Messungen des Oberflächen-EMGs vom oberen Anteil des Musculus trapecius zu klären. Ziel der Studie war die Messung der Muskelanspannung während willkürlicher Entspannung sowie während geistiger und körperlicher Aufgaben und die Messung der Dauer bis zur Entspannung nach geistiger und körperlicher Anstrengung. Die Frage war, ob Patienten mit Migräne eine konstant erhöhte Anspannung der Halsmuskulatur zeigen oder - wenn dies nicht der Fall ist, ob sie unter geistigem oder körperlichen Stress häufiger mit einer Muskelanspannung reagieren. Zudem wollten die Autoren klären, ob Aktivitäten entfernter Körperteile, z. B. der unteren Extremität bei Migränebetroffenen eine verstärkte Aktivierung der $\mathrm{Na}$ ckenmuskulatur zur Folge haben.

In die Studie wurden Personen mit Migräne ohne und mit Aura sowie migränefreie Kontrollen eingeschlossen. Den Teilnehmern wurde der Patient Health Questionnaire und der Neck Disability Index vorgelegt. Migränepatienten füllten zudem den MIDAS (Migraine Disability Assessment) aus. Das Experiment bestand aus sieben Bedingungen abwechselnd mit Entspannungszeiten und bestand somit aus insgesamt 15 Blöcken. Die ersten drei Blöcke waren mental belastend: die Teilnehmer mussten so schnell wie möglich rückwärts zählen, nämlich (1) von 100 in Schritten von 9, (2) von 80 in Schritten von 7 und (3) von 60 in Schritten von 4. Vier Blöcke beinhalteten körperliche Aktivität der unteren Extremitäten: Druck mit dem rechten / linken Fuß auf eine Körperwaage und Halten eines Gewichts von mindestens 27 kg und Streckung des rechten / linken Knies gegen Widerstand für 30 s. Die Muskelaktivität wurde mittels Oberflächen-EMG vom oberen Anteil des Musculus trapecius beidseits gemessen.

Untersucht wurden 28 migränefreie Personen, 43 Patienten mit episodischer Migräne und 31 Patienten mit chronischer $\mathrm{Mi}$ gräne. Während des Experiments berichteten $32 \%$ der Personen mit episodischer
Migräne und 65\% der Teilnehmer mit chronischer Migräne über Kopfschmerzen. Im EMG war die Muskelaktivität in allen drei Gruppen während der geistigen und körperlichen Aufgaben höher. Mit einer einzigen Ausnahme (dem Rückwärtszählen ab 100), fand sich für keine der Aufgaben ein Unterschied zwischen migränefreien Teilnehmern und Patienten mit episodischer oder chronischer Migräne. Ebenso wenig hatten die Bedingung „Ruhe“ und das Vorhandensein akuter Kopfschmerzen während des Experiments einen Einfluss auf die mittels Oberflächen-EMG gemessene Muskelaktivität. Auch die Zeit bis zur Entspannung nach geistiger und körperlicher Anstrengung war in den drei Studiengruppen vergleichbar.

Die Autoren schließen aus diesen Ergebnissen, dass die häufig beobachteten Nackenschmerzen vor oder während einer Migräneattacke nicht auf eine erhöhte Trapeziusaktivität während Ruhe, mentalem Stress und körperlicher Aktivität oder auf anhaltende Muskelaktivität zurückgeführt und nicht als ständig zugrunde liegender Auslöser erachtet werden sollten, sondern vielmehr ein Migränesymptom darstellen.

\section{Kommentar}

Die Arbeit weist insofern einen erheblichen methodischen Mangel auf, als die Schlussfolgerung (dass Nackenschmerzen bei Migräne nicht auf eine erhöhte Trapeziusaktivität zurückzuführen wären) aus den erhobenen Daten nicht ableitbar ist. Das Vorhandensein von Nackenschmerzen wurde während des Experiments nämlich gar nicht erhoben. Dieser Umstand wird weder im Abschnitt „Limitationen“ angeführt, noch wird die vorhandene Literatur zur Korrelation zwischen stress-induzierten Schmerzen und EMG-Befunden diskutiert. Die einzig zulässige Schlussfolgerung wäre die im Titel getroffene gewesen, die allerdings insofern sprachlich ungenau ist, als eine mittels EMG ermittelte veränderte Muskelaktivität kein „Symptom“ ist.

Bezüglich des Studienziels findet sich in "Abstract" und „Background“ insofern eine Diskrepanz, als in Ersterem als Studienziel dezidiert die Klärung des Zusammenhangs zwischen Nackenschmerzen und (mittels EMG) gemessener Muskelaktivie- rung angeführt wird, während in Zweitem die oben angeführte - bezogen auf die erhobenen Daten - korrekte Formulierung gewählt wurde (ob Patienten mit Migräne eine konstant erhöhte Anspannung der Halsmuskulatur zeigen oder - wenn dies nicht der Fall ist, ob sie unter geistigem oder körperlichen Stress häufiger mit einer Muskelanspannung reagieren). Eine offensichtlich falsche Formulierung findet sich auch in den Ausschlusskriterien, in denen „nicht jünger als 18 Jahre“ und „nicht an einer neurologischen, psychiatrischen oder Schmerzerkrankung leidend" erwähnt sind.

Ein Rationale für die von den Autoren als „wichtig“ erachtete Frage, ob Aktivität in entfernten Körperteilen eine höhere Aktivität in der Nackenmuskulatur zur Folge hat, wird den Lesern vorenthalten. Positiv zu bewerten ist das Studiendesign mit mehreren Belastungstests und einer angemessenen Anzahl von Patienten. Die Studie beleuchtet den Zusammenhang zwischen EMG-Befunden und Migräne trägt aber nicht zur Klärung des Zusammenhangs zwischen $\mathrm{Na}$ ckenschmerzen und Migräne bei.

Christian Wöber, Wien

\section{Migräne mit Aura und Vorhofflimmern}

\footnotetext{
*** Sen S et al. Migraine with visual aura: a risk factor for incident atrial fibrillation. Neurology 2018; 91: e1-e9.
}

Die epidemiologische Studie findet bei Patienten mit Migräne mit Aura ein leicht erhöhtes Risiko für die Entwicklung eines Vorhofflimmerns über einen Zeitraum von 20 Jahren. Sie schlägt vor, dass dies die erhöhte Inzidenz von Schlaganfällen in dieser Gruppe erklären könnte.

\section{Zusammenfassung}

Der Zusammenhang zwischen Migräne (insbesondere mit Aura) und kardiovaskulären Erkrankungen wird seit vielen Jahren diskutiert. Immer wieder tauchen Studien auf, die einen signifikanten, wenn auch absolut nur geringfügigen, Zusammenhang belegen, so etwa bei Schlaganfall und Herzinfarkt. Die aktuelle Studie beschäftigt sich 
mit der Frage, ob Menschen mit Migräne ein höheres Risiko haben, ein Vorhofflimmern zu entwickeln. Dies ist von Bedeutung, da eine erhöhte Rate von Vorhofflimmern die erhöhte Rate an Schlaganfällen erklären könnte. In die Studie wurden 11939 gesunde Patienten eingeschlossen, die 1993 bis 1995 nach ihrer Migränegeschichte befragt worden waren. Im Jahr 2013 wurde dann ermittelt, wer Vorhofflimmern entwickelt hatte. In der Migränegruppe waren dies $15 \%$ und in der Kontrollgruppe ohne Kopfschmerzen waren dies $17 \%$, dies war ohne Adjustierung sogar signifikant, d. h. Migräne war also ein Schutzfaktor gegen Vorhofflimmern. Nach Adjustierung für verschiedene Faktoren blieb übrig, dass Migräne mit visueller Aura einen signifikanten Risikofaktor für die Entwicklung von Vorhofflimmern darstellte, es hatten in dieser Gruppe nämlich 19\% $(n=80)$ ein Vorhofflimmern entwickelt. In verschiedenen Modellberechnungen wurde dann ermittelt, ob das Vorhofflimmern mit Migräne einen Risikofaktor für einen kardioembolischen Schlaganfall darstellte. Hierbei ergab sich ein Zusammenhang zwischen Vorhofflimmern und Schlaganfall, aber nicht zwischen Migräne mit visueller Aura und Schlaganfall, wenn für Vorhofflimmern kontrolliert wurde. Zusammenfassend glauben die Autoren, dass der bereits beschriebene Zusammenhang zwischen Migräne mit Aura und Schlaganfall möglicherweise auf die erhöhte Rate von Vorhofflimmern in dieser Gruppe zurückzuführen ist. Sie wiesen u. a. darauf hin, dass in Fallberichten häufiger schon das Auftreten von Vorhofflimmern während einer Migräneattacke beschrieben worden ist.

\section{Kommentar}

Der Zusammenhang zwischen kardio- und zerebrovaskulären Erkrankungen und Migräne ist in den letzten Jahren so intensiv untersucht worden, dass dieses Thema ganz oben in der epidemiologischen Diskussion über Migräne steht und auch von Patienten zunehmend wahrgenommen wird. Da ist es auf der einen Seite natürlich zu begrüßen, wenn neue Befunde vorgelegt werden, auf der anderen Seite sollte man sehr genau die Limitierungen und Inkonsistenzen beachten, die die Studien der letzten Jahre gebracht haben. Die hier vorgelegte Studie legt nahe, einen kausalen Zusammenhang zwischen Migräne mit Aura und Schlaganfall gefunden zu haben. Hierbei müssen aber zahlreiche Bedenken angebracht werden. Erst einmal muss beachtet werden, dass die Gruppe von Patienten mit diesem Zusammenhang bei $n=33$ lag. Dann zeigte diese Studie nicht den Zusammenhang zwischen Migräne mit Aura und Schlaganfall im Allgemeinen, der ja in zahlreichen vorherigen Studien gezeigt worden ist. Weiterhin ist die Definition der visuellen Aura anzuzweifeln. Es wurde nämlich nur gefragt, ob die Patienten vor den Kopfschmerzen Streifen, Blitze oder wellenförmige Linien gesehen hatten. Dies galt schon als Aura, ohne Beachtung der Zeitkriterien für eine Aura und ohne Beachtung der Aura ohne Kopfschmerzen. Dann war die Diagnose Vorhofflimmern nur gestützt auf ein EKG oder auf Krankenhausberichte. Die tatsächliche Inzidenz konnte natürlicherweise nicht ermittelt werden, da sie mehrere Langzeit-EKG oder implantierte Event Recorder vorausgesetzt hätte. Schließlich wurde nicht kontrolliert für das Vorliegen eines PFO, obwohl dies bei Migräne mit Aura eine signifikant erhöhte Prävalenz zeigt. Zusammenfassend zeigt diese Studie also, wie vorsichtig man naheliegende Interpretationen von epidemiologischen Studien beurteilten muss, insbesondere bei der so sensiblen Frage, ob Migräne einen Risikofaktor für vaskuläre Erkrankungen darstellt oder nicht.

Stefan Evers, Coppenbrügge

\section{Die neue europäische Leitlinie zur idiopathi- schen intrakraniellen Hypertension ist erschienen}

**** Hoffmann J et al. European Headache Federation guideline on idiopathic intracranial hypertension. The Journal of Headache and Pain 2018; 19: 93.

\section{Hintergrund}

Zu den gesicherten pathophysiologischen Mechanismen bei der Entstehung von Kopfschmerzen gehört neben entzündlichen neurovaskulären Prozessen vor allem auch die intrakranielle Druckerhöhung. In den letzten Jahren hat sich die Wissensba- sis zur idiopathischen intrakraniellen Hypertension durch verschiedene Studien erheblich erweitert. Es gibt neue Erkenntnisse über wegweisende bildgebende Befunde und endlich auch Therapiestudien zur Gewichtsreduktion und zur Behandlung mit Acetazolamid.

\section{Zusammenfassung und Kommentar}

Die vorliegende Arbeit der Europäischen Kopfschmerzgesellschaft stellt die aktuellen Erkenntnisse zu dem Krankheitsbild zusammen und liefert eine umfassende Übersicht über das klinische Bild, typische Befunde der apparativen Zusatzdiagnostik sowie der medikamentösen und operativen Therapie der idiopathischen intrakraniellen Hypertension. Sekundäre Syndrome, die zu einer intrakraniellen Hypertension führen, bleiben in der Arbeit weitgehend unberücksichtigt. Die Arbeit ist besonders lesenswert, weil sie eine Brücke in das Fach der Ophthalmologie schlägt und mit anschaulichem Bildmaterial besondere Techniken zur Bestimmung des Gesichtsfeldes sowie den Stellenwert von OCT-Untersuchungen beleuchtet und damit eine gute Voraussetzung für ein besseres Verständnis von den ophthalmologischen Zusatzbefunden liefert, die für die Verlaufsbeurteilung der Betroffenen essentiell sind. Leider werden die bildgebenden Befunde nicht in derselben Ausführlichkeit vorgestellt. Die Arbeit wertet vor allem die unterschiedlichen Behandlungsoptionen kritisch und bietet damit eine gute Wissensbasis für die Therapie von Patientinnen und Patienten mit einer intrakraniellen Hypertension. Manche Aspekte, die bei der idiopathischen intrakraniellen Hypertension eine Rolle spielen können wie z. B. psychische Komorbiditäten und die Komorbidität mit anderen primären Kopfschmerzerkrankungen, insbesondere mit der Migräne, hätte die Arbeit vielleicht etwas deutlicher herausstellen können, da diese Aspekte vor allem bei der Betreuung von chronischen Kopfschmerzpatienten von besonderer Bedeutung sind. Jeder Facharzt, der Patienten mit chronischen Kopfschmerzen betreut, sollte bei unzureichendem Ansprechen auf die Therapie an die Differentialdiagnose einer idiopathischen intrakraniellen Hypertension denken und ggf. eine LP mit Druckmes- 
sung durchführen, zumal der Nachweis eine Stauungspapille für den Einzelnen schwierig sein kann und nicht bei allen betroffenen Patienten tatsächlich auch eine Stauungspapille vorliegt. Die Arbeit ist insgesamt sehr ausgewogen und lesenswert. Im Aufbau entspricht sie eher einer Übersichtsarbeit denn einer Leitlinie.

Stefanie Förderreuther, München

\section{Differenzialdiagnose RCVS und primäre ZNS-Vaskulitis}

\footnotetext{
*** de Boysson $\mathrm{H}$ et al. Primary angiitis of the CNS and reversible cerebral vasoconstriction syndrome: A comparative study. Neurology 2018; 91: e1468e-1478.
}

Beginn mit einem akuten Donnerschlagkopfschmerz und initial unauffällige MRT-Bildgebung sind fast beweisend für ein RCVS.

\section{Zusammenfassung}

Die Arbeitsgruppe aus Paris, die Fallserien und Übersichtsartikel zum reversiblen zerebralen Vasokonstriktions-Syndrom (RCVS) veröffentlicht, vergleicht in der Arbeit die klinische Manifestation, Liquor- und Bildgebungsbefunde des RCVS mit denen bei primärer Angiitis des ZNS (PACNS). Dabei werden die Daten von 110 Patienten der französischen nationalen „COVAC“-Studie (mit PACNS) und 173 RCVS-Patienten, die die Autoren seit 2004 in ihrem Zentrum gesehen haben, verglichen.

Die Diagnose einer PACNS wurde dann als gesichert angesehen, wenn die Klinik mit Kopfschmerz, fokalen neurologischen Defiziten und/oder neurokognitiven Veränderungen sowie Anfällen vereinbar mit der Diagnose PACNS war. Darüber hinaus wurden entsprechende Auffälligkeiten in der Bildgebung, gegebenenfalls Auffälligkeiten in der Biopsie (wobei nur $32 \mathrm{~Pa}$ tienten biopsiert wurden), sowie der Ausschluss einer infektiösen (Hepatitis, Tuberkulose, HIV) oder rheumatischen Genese (Serologie) gefordert, und die Patienten mindestens 6 Monate nachverfolgt, um so ein RCVS auszuschließen. Für die Diagnose eines RCVS wurde ein akuter Beginn mit
Kopfschmerzen, bildgebend der Nachweis von Vasospasmen in zwei Gefäßgebieten sowie deren Remission innerhalb von 3 Monaten gefordert. Durch den Vergleich ließen sich eindeutige Unterschiede herausarbeiten, die eine frühzeitige Diagnosestellung ermöglichen. Für ein RCVS spricht ein weibliches Geschlecht, eine vorbestehende Migräne, die Einnahme von vasoaktiven Substanzen (Cannabis, SSRI, vasoaktive Nasensprays), akuter Beginn mit einem (Donnerschlag-)Kopfschmerz (99\%, zum Teil mit einem Auslöser wie sexuelle Aktivität, emotionaler Stress, Anstrengung) und eine initial unauffällige Bildgebung (69\% der Fälle). Für eine PACNS sprechen neurologische Defizite, auffällige Bildgebung (in allen Fällen) und Liquorveränderungen bei Erstvorstellung. Weitere interessante Unterschiede waren der im Mittel höhere Blutdruck und das häufigere Vorliegen von PRES-ähnlichen MRT-Auffälligkeiten (vasogenes Ödem) beim RCVS. Nur das RCVS trat postpartal auf, kein Patient mit PACNS wurde in der postpartalen Periode diagnostiziert. Anfälle, Aphasie sowie jegliche anderen fokalen Defizite waren häufiger bei PACNS. Ein stark erhöhtes Liquoreiweiß kommt nur beim PACNS vor.

\section{Kommentar}

Die Stärke der Arbeit ist, dass zwei relativ große Kohorten, die sehr gut klinisch und auch durch Bildgebung charakterisiert sind, verglichen werden. So können klinisch wichtige Anhaltspunkte gefunden werden, die anhand weniger Punkte eine rasche Differentialdiagnose ermöglichen. Die Befunde sind aber an sich nicht neu und Neurologen, die regelmäßig mit Notfallpatienten konfrontiert werden, sind diese Befunde bekannt. So kann eine PACNS ohne bildgebende Auffälligkeiten (Infarkte, Blutungen) nicht diagnostiziert werden, auch die häufig auffälligen Liquorbefunde sind bekannt. Ein wichtiger Punkt ist, dass ein wiederholter Donnerschlagkopfschmerz nur bei einem RCVS zu beobachten ist. Leider geht die Arbeit nicht auf mögliche klinische Unterschiede zwischen einer zerebralen Kleingefäßvaskulitis und einer Vaskulitis der großen Gefäße ein. Aus Sicht des Rezensenten ist die Abgrenzung zwischen zerebraler Angiitis und zerebraler Angiopathie (z. B. Moya-Moya) im Einzelfall viel schwieriger, als die Abgrenzung von PACNS zu RCVS, die auf dem Boden der beschriebenen Unterschiede relativ leicht ist.

Andreas Straube, München

INFOR M ATION
***** Exzellente Arbeit, die bahn-
brechende Neuerungen
beinhaltet oder eine ausge-
zeichnete Übersicht bietet
Gute experimentelle oder
klinische Studie
Gute Studie mit allerdings
etwas geringerem Innovati-
onscharakter
Studie von geringerem
klinischen oder experimentel-
len Interesse und leichteren
methodischen Mängeln
Studie oder Übersicht mit
deutlichen methodischen
oder inhaltlichen Mängeln
der
des jeweiligen Autors dar, nicht eine
sche Migräne- und Kopfschmerzge-
rellschaft.
Die Kopfschmerz-News werden
betreut von: Priv.-Doz. Dr. Ruth
Ruscheweyh, Klinik und Poliklinik für
Neurologie, Klinikum der Universität
München, Marchioninistr. 15, 81377
München, Tel. 089/440073907, ruth.

